\title{
Efficacy of Various Intracanal Medicaments in Human Primary Teeth with Necrotic Pulp against Candida Biofilms: An in vivo Study
}

\author{
1 Jophie V Paikkatt, ${ }^{2}$ Sheela Sreedharan, ${ }^{3}$ Beena Philomina, ${ }^{4}$ VP Kannan, ${ }^{5}$ Madhu Santhakumar, ${ }^{6}$ TV Anupam Kumar
}

\begin{abstract}
Background: Candida has been associated with cases of secondary and persistent root canal infections. The purpose of this study was to evaluate and compare the effectiveness of commonly used intracanal medicament against Candida biofilms found in root canals of human primary teeth with necrotic pulp.

Materials and methods: Pulp canals of 45 single-rooted primary maxillary anterior teeth with pulp necrosis in 34 children were included in the study. They were divided into three groups of 15 samples each - group I: $\mathrm{Ca}(\mathrm{OH})_{2}$ (calcium hydroxide); group II: $1 \%$ chlorhexidine gel (CHX); and group III: $1 \%$ metronidazole gel. Bacterial count was obtained from each tooth at two different stages: (1) after instrumentation, and (2) after placement of the medication. Statistical analysis using the Statistical Package for the Social Sciences version 10.0 software program (Inc., Chicago, IL, USA) with Wilcoxon signed rank test after grouping the samples was performed.
\end{abstract}

Results: $\mathrm{Ca}(\mathrm{OH})_{2}, 1 \% \mathrm{CHX}$ gel, and $1 \%$ metronidazole gel were ineffective in completely eliminating Candida biofilms from root canal of human primary teeth with necrotic pulp.

Conclusion: None of the commonly used intracanal medicaments, i.e., $\mathrm{Ca}(\mathrm{OH})_{2}, 1 \% \mathrm{CHX}$ gel, and $1 \%$ metronidazole gel, was effective in completely eliminating Candida biofilm from root canal of human primary teeth with necrotic pulp. Ineffectiveness of these medicaments against Candida has opened new door of research regarding the use of suitable intracanal medicaments against single and multispecies biofilms.

Keywords: Calcium hydroxide, Candida, Chlorhexidine, Metronidazole.

\footnotetext{
${ }^{1}$ Reader, ${ }^{2,4}$ Professor and Head, ${ }^{3}$ Professor, ${ }^{5,6}$ Associate Professor ${ }^{1}$ Department of Pedodontics, MES Dental College, Perinthalmanna Kerala, India

${ }^{2}$ Department of Pedodontics, Government Dental College Thiruvananthapuram, Kerala, India

${ }^{3}$ Department of Microbiology, Government Medical College Kozhikode, Kerala, India

${ }^{4-6}$ Department of Pedodontics, Government Dental College Kozhikode, Kerala, India
}

Corresponding Author: Jophie V Paikkatt, Reader, Department of Pedodontics, MES Dental College, Perinthalmanna, Kerala India, Phone: +914933249100, e-mail: paikkattjophie@gmail.com
How to cite this article: Paikkatt JV, Sreedharan S, Philomina B, Kannan VP, Santhakumar M, Kumar TVA. Efficacy of Various Intracanal Medicaments in Human Primary Teeth with Necrotic Pulp against Candida Biofilms: An in vivo Study. Int J Clin Pediatr Dent 2017;10(1):45-48.

Source of support: Nil

Conflict of interest: None

\section{INTRODUCTION}

The presence of fungi in the root canals was first reported by Grossman, ${ }^{1}$ who found fungal evidence in $17 \%$ of evaluated sample. Fungi have been isolated from root canals of teeth with pulpal necrosis and apical periodontitis ${ }^{2,3}$ and have been reported as a potential cause of endodontic failure in root-filled teeth. ${ }^{4,5}$

Candida is the most commonly isolated yeast genus from infected root canal and is potentially pathogenic. ${ }^{5}$ The mechanisms believed to be involved in pathogenesis are (a) adaptability to a variety of environmental conditions; (b) adhesion to a variety of surfaces; (c) production of hydrolytic enzymes; (d) morphologic transition; (e) biofilm formation; and (f) evasion and immunomodulation of host defence. ${ }^{6}$

Hence, the elimination of Candida from root canals of infected teeth leads to successful treatment.

Endodontic instrumentation cannot effectively eliminate the microflora from root canals of primary teeth mechanically owing to their anatomic complexity and need of patient cooperation. ${ }^{7}$ Hence, dependence on intracanal medicament for endodontic success becomes more important. Several medicaments have been attempted in root canals of permanent teeth with their respective advantages/disadvantages; however, there is a paucity of literature related to efficacy of various medication used in primary teeth.

Calcium hydroxide $\left[\mathrm{Ca}(\mathrm{OH})_{2}\right]$, is the "gold standard" endodontic medicament used widely to eliminate microbes that survive instrumentation. ${ }^{8,9}$ A 1\% chlorhexidine $(\mathrm{CHX})$ gel is an alternate medicament used in endodontic practice as an effective antimicrobial means for disinfecting the root canal. ${ }^{9,10}$ Medication was selected in gel form as they have both antimicrobial and lubricating actions during instrumentation. ${ }^{10}$ 
Because of increasing interest regarding the role of Candida biofilms in root canal infections and its association with failed endodontic therapy and recent reports of fungal resistance against some medicaments, the purpose of this clinical study was to find the antimicrobial efficacy of commonly used intracanal medicaments against Candida biofilms in root canals of human primary teeth with necrotic pulp.

\section{MATERIALS AND METHODS}

Eligible participants for this clinical trial were selected from patients of both genders aged 4 to 6 years that had been referred for dental treatment at Pediatric Dentistry clinic.

Fulfillment of following inclusion criteria was required for patient enrollment, based on clinical and radiographic examination-asymptomatic maxillary incisor teeth (both central or laterals) with confirmed pulpal necrosis owing to caries (with or without periapical lesion), but with sufficient coronal structure to permit isolation of operative field with rubber dam, less than two-thirds of root resorption, presence or not of fistula, mobility degree 0 or 1 , and no periodontal pocket. If present, the periapical lesion should not be invading the follicles of germ of permanent successor. The 34 patients, who met all of these inclusion criteria, were enrolled, providing a total sample of 45 teeth. The study purposes were fully explained to parents/guardians, who signed a written informed consent form. The research protocol was received and approved by institutional Research Ethics Committee.

\section{Medicaments Tested}

- $\mathrm{Ca}(\mathrm{OH})_{2}$ paste ( $\mathrm{RC}$ Cal, Prime Dental Product, India)

- $1 \%$ CHX gel (Hexigel, ICPA Ltd., India)

- $1 \%$ metronidazole gel (Metogyl DG gel, Unique Pharmaceuticals, India).

\section{Methods of the Study}

For every appointment, three teeth were selected from patients who met the inclusion criteria. The three maxillary incisors were divided into three experimental groups as described below:

1. Group $\mathrm{I}-\mathrm{Ca}(\mathrm{OH})_{2}$ paste

2. Group II- $1 \%$ CHX gel

3. Group III-1\% metronidazole gel

After isolation with a rubber dam, the teeth were disinfected with 30\% hydrogen peroxide and 10\% tincture iodine. ${ }^{11}$ The pulp chambers were opened using aseptic conditions. Airotor with sterile cooled water and highspeed diamond round burs were used. After confirming the working length, instrumentation was performed $1 \mathrm{~mm}$ above the apices with Hedstroem $(\mathrm{H})$ files, upto size 50. After the mechanical preparation and irrigation, a sterile, size 20 paper point was introduced into the length of the root canals for the initial microbiological sampling. The paper point was placed for 60 seconds in the canal and then immediately transferred to a sterile test tube. The test tubes containing paper point sample were taken to the Department of Microbiology for processing within 1 hour. ${ }^{12}$

After collecting the sample, the root canals were dried and medicaments placed depending on the group. All medicaments were applied with a syringe and a 26-gauge needle. Subsequently, a sterile cotton pellet was placed at the entrance and the cavities were temporarily sealed with zinc oxide eugenol cement.

For microbiological procedures, the paper point samples were rolled in blood agar plates, and the plate was incubated in an incubator for 48 hours at $37^{\circ} \mathrm{C}$.

Candida was seen as a nonhemolytic dry white colony; the colony morphology was studied and count of Candida was determined as colony-forming unit (CFU) depending on growth on number of streaks. The growth of Candida was further confirmed by Gram staining. Candida was seen as oval budding yeast 2 to $6 \mu \mathrm{m}$ in diameter. In order to evaluate the effect of the medicaments against Candida, the patients were recalled after 2 weeks. ${ }^{7}$ After irrigation with saline, a second microbiological sampling was carried out with sterile paper points in the root canals as described earlier.

After the microbiological sampling, all the root canals were filled with zinc oxide eugenol paste and all treated teeth were given fiber-reinforced post and crown.

Statistical analysis was carried out using the Statistical Package for the Social Sciences version 10.0 software program (Inc., Chicago, Illinois, USA) with Wilcoxon signed rank test after grouping the samples.

\section{RESULTS}

A total of 45 teeth were studied. They included three groups of 15 teeth each. Group I was medicated with $\mathrm{Ca}(\mathrm{OH})_{2}$, group II with $1 \% \mathrm{CHX}$ gel, and group III with $1 \%$ metronidazole gel.

Table 1 outlines that $\mathrm{Ca}(\mathrm{OH})_{2}, 1 \% \mathrm{CHX}$ gel, and $1 \%$ metronidazole gel have no statistically significant action on Candida.

After taking the average count of Candida in CFUs after various medications, Graph 1 shows that none of the commonly used medicaments, i.e., $\mathrm{Ca}(\mathrm{OH})_{2}, 1 \% \mathrm{CHX}$ gel, or $1 \%$ metronidazole gel, was able to completely eradicate Candida from the root canal system. 
Table 1: Effect of various medicaments on Candida

\begin{tabular}{lccc}
\hline & Candida after $\mathrm{Ca}(\mathrm{OH})_{2}$ medication & Candida after CHX vs & $\begin{array}{c}\text { Candida after metronidazole } \\
\text { vs Candida after BMP }\end{array}$ \\
\hline Vs Candida after BMP & -1.089 & -0.577 & -1.633 \\
b Asymp. sig. (two-tailed) & 0.276 & 0.564 & 0.102 \\
& $\mathrm{NS}$ & $\mathrm{NS}$ & $\mathrm{NS}$ \\
\hline
\end{tabular}

${ }^{b}$ Wilcoxon signed rank test; NS: Nonsignificant; BMP: Biomechanical preparation

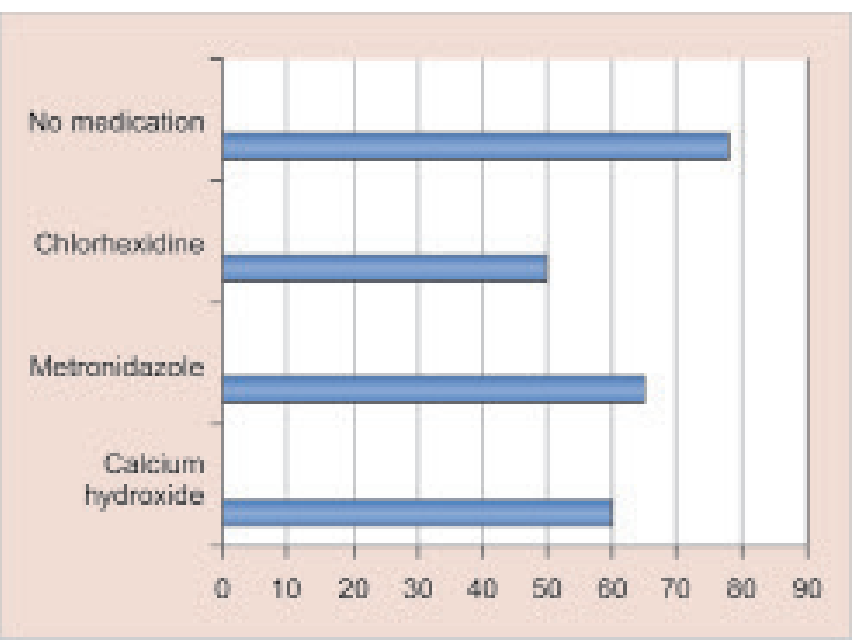

Graph 1: Average count of Candida in CFU after various medicaments

\section{DISCUSSION}

The antimicrobial effectiveness of $1 \% \mathrm{CHX}$ gel, $\mathrm{Ca}(\mathrm{OH})_{2}$, and $1 \%$ metronidazole gel is well investigated in vitro. It appeared that they have wide antimicrobial activity against microorganisms isolated from root canal system. However, the results found from in vitro study cannot be directly extrapolated to clinical situations because of optimum contact between medication and dentin under in vitro condition and because of variable imposed by clinical situation and not reproduced under experimental model. Hence, the present clinical study was undertaken after taking into consideration the above objective.

Candida in nature rarely exists in planktonic state, but is organized in biofilm structures, which is a complex community, composed of great variety of organisms with different ecological requirement and pathogenic potential. Biofilm growth is a continuous process that goes through various stages from young to mature, structurally complex biofilm. ${ }^{13}$ In necrotic pulp with harsh ecological milieu, it is likely that the physiological state of biofilm is closest to mature, hence making it resistant to various antimicrobial agents. The protective mechanism underlying biofilm antimicrobial resistance is not fully understood, although several mechanisms have been proposed..$^{14}$ These mechanisms include physical or chemical diffusion barrier to antimicrobial penetration into the biofilm, slow growth of biofilm owing to nutrient limitation, activation of general stress response, and emergence of biofilm-specific phenotype. ${ }^{15}$

The CHX is a cationic bisguanide with broad antimicrobial activity, low mammalian toxicity, and strong affinity to binding to dentin and mucous membrane. The CHX molecules react with negatively charged groups on the cell surface, causing an irreversible loss of cytoplasmic constituents, membrane damage, and enzyme inhibition. It is likely that ionic interaction occurs between the positively charged $\mathrm{CHX}$ molecules and negatively charged extracellular matrix. This ionic interaction is understood to reduce the diffusion of fluorescent probes within biofilms by about 50 fold, thus explaining the possible mechanism of Candida resistance to $1 \% \mathrm{CHX}$ gel. ${ }^{16}$

As seen in previous studies, Candida was resistant to $\mathrm{Ca}(\mathrm{OH})_{2}$. Candida survives in a wide range of $\mathrm{pH}$ values; the alkalinity of $\mathrm{Ca}(\mathrm{OH})_{2}$ may not have any effect on Candida. In addition, $\mathrm{Ca}(\mathrm{OH})_{2}$ readily displaces $\mathrm{Ca}^{++}$ion necessary for growth and morphogenesis of Candida. ${ }^{8,9}$

Metronidazole is bactericidal against most anaerobes that contain electron transport components, such as ferredoxin, which donates electrons to metronidazole, forming highly reactive nitroradical anions that kill susceptible organisms by a radical-mediated mechanism. A $1 \%$ metronidazole gel in this study has not been effective against Candida, as it is active against strict anaerobes, but is ineffective against facultative anaerobes. ${ }^{17,18}$

\section{CONCLUSION}

On the basis of observations made during the course of the present study, the following conclusions were drawn.

Firstly, $\mathrm{Ca}(\mathrm{OH})_{2}, 1 \% \mathrm{CHX}$ gel, and $1 \%$ metronidazole gel, which are commonly used intracanal medicaments, were found to be ineffective in completely eliminating Candida biofilms from root canals of primary teeth with necrotic pulp. Secondly, ineffectiveness of these medicaments against Candida has opened new door of research regarding the use of intracanal medicaments on single and multispecies biofilms.

\section{REFERENCES}

1. Grossman LI. Root canal therapy. 3rd ed. London: Henry Kimpton; 1952. 
2. Ferrari PHP, Cai S, Bombana AC. Effect of endodontic procedures on Enterococci, enteric bacteria \& yeast in primary endodontic infections. Int Endod J 2005 Jun;38(6):372-380.

3. Siqueira JF Jr, Sen BH. Fungi in endodontic infection. Oral Surg Oral Med Oral Pathol Oral Radiol Endod 2004 May;97(5): 632-641.

4. Pinheiro ET, Gomes BP, Ferraz CC, Souza EL, Teixeira FB, Souza-Filho FJ. Microorganisms from canals of root filled teeth with periapical lesions. Int Endod J 2003 Jan;36(1):1-11.

5. WaltimoTMT,Sen BH,MeurmanJH,OrstavikD,HaapasaloMP. Yeast in apical periodontitis. Crit Rev Oral Biol Med 2003;14(2): 128-137.

6. Sinde PT, Olsen I, Debelian GJ, Tronstand J. Microbiota of periapical lesions refractory to endodontic therapy. J Endod 2002 Apr;28(4):304-310.

7. Bystrom A, Sundqvist G. Bactriological evaluation of efficacy of mechanical root canal instrumentation in endodontic therapy. Scand J Dent Res 1981 Aug;89(4):321-328.

8. de Rezende GP, da Costa LR, Pimenta FC, Baroni DA. In vitro antimicrobial activity of endodontic paste with propolis extracts and calcium hydroxide: a preliminary study. Braz Dent J 2008 Jan;19(4):301-305.

9. Basrani B, Ghanem A, Tjaderhane L. Physical and chemical properties of chlorhexidine and calcium hydroxide containing medications. J Endod 2004 Jun;30(6):413-417.

10. Athanassiadis B, Abbott PV, Walsh LJ. The use of calcium hydroxide, antibiotics and biocides as antimicrobial medicaments in endodontics. Aust Dent J 2007 Mar;52(Suppl 1): S64-S82.
11. Moller AJ. Microbiological examination of root canals and periapical tissues of human teeth. Methodological studies. Odontol Tidskr 1966 Dec;74(5):1-380.

12. Forbes AB, Daniel FS, Weissfeld AS. Upper respiratory infection and other infections of oral cavity and neck. In: Bailey, Scott, editors. Textbook of diagnostic microbiology. 11th ed. Missouri: Mosby Publishers; 2002. p. 903.

13. Stewart PS, Rayner J, Roe F, Rees WM. Biofilm penetration and disinfection efficacy of alkaline hypochlorite and chlorosulfamates. J Appl Microbiol 2001 Sep;91(3):525-532.

14. Mah TF, O'Toole GA. Mechanisms of biofilm resistance to antimicrobial agents. Trends Microbiol 2001 Jan;9(1):34-39.

15. Stewart PS. Diffusion in biofilms. J Bacteriol 2003 Mar;185(5): 1485-1491.

16. Golot E, Georges P, Brin A, Fontaine-Aupart MP, BellonFontaine MP, Briandet R. Heterogeneity of diffusion inside microbial biofilms determined by fluorescence correlation spectroscopy using two photon excitation. Photochem Photobiol 2002 Jun;75(6):570-578.

17. Siquiera JF, Uzeda MD. Intracanal medicaments: evaluation of the antibacterial effects of chlorhexidine, metronidazole and calcium hydroxide associated with three vehicles. J Endod 1997 Mar;23(3):167-169.

18. Kirthikadatta J, Indira R, Dorothy Kalyani AL. Disinfection of dentinal tubules with $2 \%$ chlorhexidine, $2 \%$ metronidazole, bioactive glass when compared with calcium hydroxide as intracanal medicaments. J Endod 2007 Dec;33(12): 1473-1476 